\section{Successful coronary angioplasty in two patients with cardiogenic shock using the Nimbus Hemopump support device}

\author{
A. Michael Lincoff, MD, Jeffrey J. Popma, MD, \\ Eric R. Bates, MD, G. Michael Deeb, MD, \\ Steven F. Bolling, MD, Joan S. Meagher, RN, \\ Ann M. Kelly RN, Richard K. Wampler, MD, and \\ John M. Nicklas, MD. Ann Arbor, Mich.
}

When standard medical therapy is employed, cardiogenic shock is associated with an in-hospital mortality rate in excess of $75 \%$. Coronary angioplasty has been shown to reduce this mortality in patients following myocardial infarction, ${ }^{1}$ but the procedure can be complicated by transient myocardial ischemia induced by prolonged balloon inflation, acute vessel closure, or arrhythmias. To avoid possible decompensation during high-risk angioplasty, intra-aortic balloon counterpulsation and percutaneous cardiopulmonary bypass have been used to help support the circulation. ${ }^{2-5}$ Although these devices can provide variable degrees of circulatory support, none directly decompress the left ventricle and unload the heart throughout the cardiac cycle. A new circulatory support device, the Nimbus Hemopump (Nimbus Medical Inc., Rancho Cordova, Calif.), decompresses the left ventricle by withdrawing blood directly from the ventricular cavity and ejecting it into the descending aorta (Fig. 1). Flow up to $3.5 \mathrm{~L} / \mathrm{min}$ is generated by an Archimedes spiral vane screw rotating at $25,000 \mathrm{rpm}$. We report two patients with cardiogenic shock who were successfully supported with the Hemopump during coronary angioplasties complicated by potentially catastrophic arrhythmias.

Case No. 1. A 48-year-old man with an extensive anterolateral myocardial infraction was treated with tissue plasminogen activator, but developed progressive congestive heart failure and hypotension over the ensuing 48 hours. Despite inotropic support, the blood pressure (BP) remained $74 / 50 \mathrm{~mm} \mathrm{Hg}$, pulmonary capillary wedge pressure (PCWP) was $37 \mathrm{~mm} \mathrm{Hg}$, and the cardiac index (CI) was 2.3 $\mathrm{L} / \mathrm{min}$. The left femoral artery was surgically exposed and the $21 \mathrm{~F}$ Hemopump was advanced into the left ventricle via a $12 \mathrm{~mm}$ Dacron graft anastomosed to the side of the vessel. With Hemopump support, BP rose to $96 / 61 \mathrm{~mm} \mathrm{Hg}$, PCWP fell to $11 \mathrm{~mm} \mathrm{Hg}$, and $\mathrm{CI}$ increased to $3.9 \mathrm{~L} / \mathrm{min}$.

From the Department of Internal Medicine, Division of Cardiology, The University of Michigan School of Medicine.

Dr. Nicklas is supported by a National Institutes of Health Clinical Investigator Award No. HL01170.

Reprint Requests: John M. Nicklas, MD, 3910 Taubman, Division of Cardiology, University of Michigan Hospital, 1500 E. Medical Center Dr., Ann Arbor, MI 48109-0366.

$4 / 4 / 22912$
Coronary arteriography was performed from the right femoral artery using the Judkins technique. An $8 \mathrm{~F}$ pigtail catheter was advanced to the ascending aorta to avoid guide wire entrapment in the Hemopump. Selective coronary arteriography demonstrated three-vessel coronary disease including a $100 \%$ occlusion of the proximal left anterior descending (LAD) coronary artery. During arteriography, the patient developed third-degree heart block with a slow ventricular escape rhythm (Fig. 2). Before placement of a pacemaker, $\mathrm{BP}$ was maintained at $50 \mathrm{~mm} \mathrm{Hg}$ with Hemopump circulatory support. Coronary angioplasty of the proximal LAD was then successfully performed using an 0.014 inch standard " $\mathrm{J}$ " guide wire (USCI Division of C.R. Bard, Billerica, Mass.) and a $3.0 \mathrm{~mm}$ balloon dilatation catheter ("Skinny", SciMed Life Systems Inc., Maple Grove, Minn.). While hemodynamically improved, the patient subsequently developed a large nonhemorrhagic frontoparietal cerebral infarction and died on the seventh hospital day.

Case No. 2. A 73-year-old man with a chronic ischemic cardiomyopathy was transferred with medically refractory cardiogenic shock. BP was $90 / 60 \mathrm{~mm} \mathrm{Hg}$, PCWP was 22 $\mathrm{mm} \mathrm{Hg}$, and CI was $1.9 \mathrm{~L} / \mathrm{min}$. Cardiac catheterization demonstrated a $70 \%$ stenosis of the left main (LM) coronary artery, $90 \%$ stenosis of the mid-LAD, $70 \%$ complex stenosis of the proximal left circumflex (LCx), and proximal occlusion of the right coronary artery. During each coronary injection BP fell to $60 / 40 \mathrm{~mm} \mathrm{Hg}$ for periods up to 30 seconds. Due to depressed left ventricular function, surgical revascularization was not attempted. The Hemopump was placed using the surgical approach described above. BP subsequently rose to $98 / 60 \mathrm{~mm} \mathrm{Hg}$, PCWP decreased to $18 \mathrm{~mm} \mathrm{Hg}$, and $\mathrm{CI}$ increased to $2.8 \mathrm{~L} / \mathrm{min}$. Coronary angioplasties of the LM, LAD, and LCx stenoses were successfully performed using the 0.014 inch "J" guide wire and a $2.5 \mathrm{~mm}$ balloon "Skinny" dilatation catheter. During coronary angioplasty, the patient had two episodes of sustained ventricular tachycardia lasting 30 seconds and 120 seconds, respectively, terminated by lidocaine injection and discontinuation of inotropic agents. A mean BP of $48 \mathrm{~mm} \mathrm{Hg}$ was maintained during each arrhythmia. Despite successful coronary revascularization, severe hemodynamic compromise persisted and the patient died on the ninth hospital day.

In the present report, successful coronary angioplasty was performed in two patients with cardiogenic shock who were supported by the Nimbus Hemopump. Systemic arterial pressure was adequately maintained in each case, even during potentially catastrophic arrhythmias. Circulatory assist devices have been advocated as adjunctive therapy during coronary angioplasty in patients with severe left ventricular dysfunction or large regions of potentially jeopardized myocardium in the distribution of the target vessels. Although circulatory assistance can be provided by a variety of devices, the Hemopump offers the unique advantage of directly decompressing the left ventricle and 


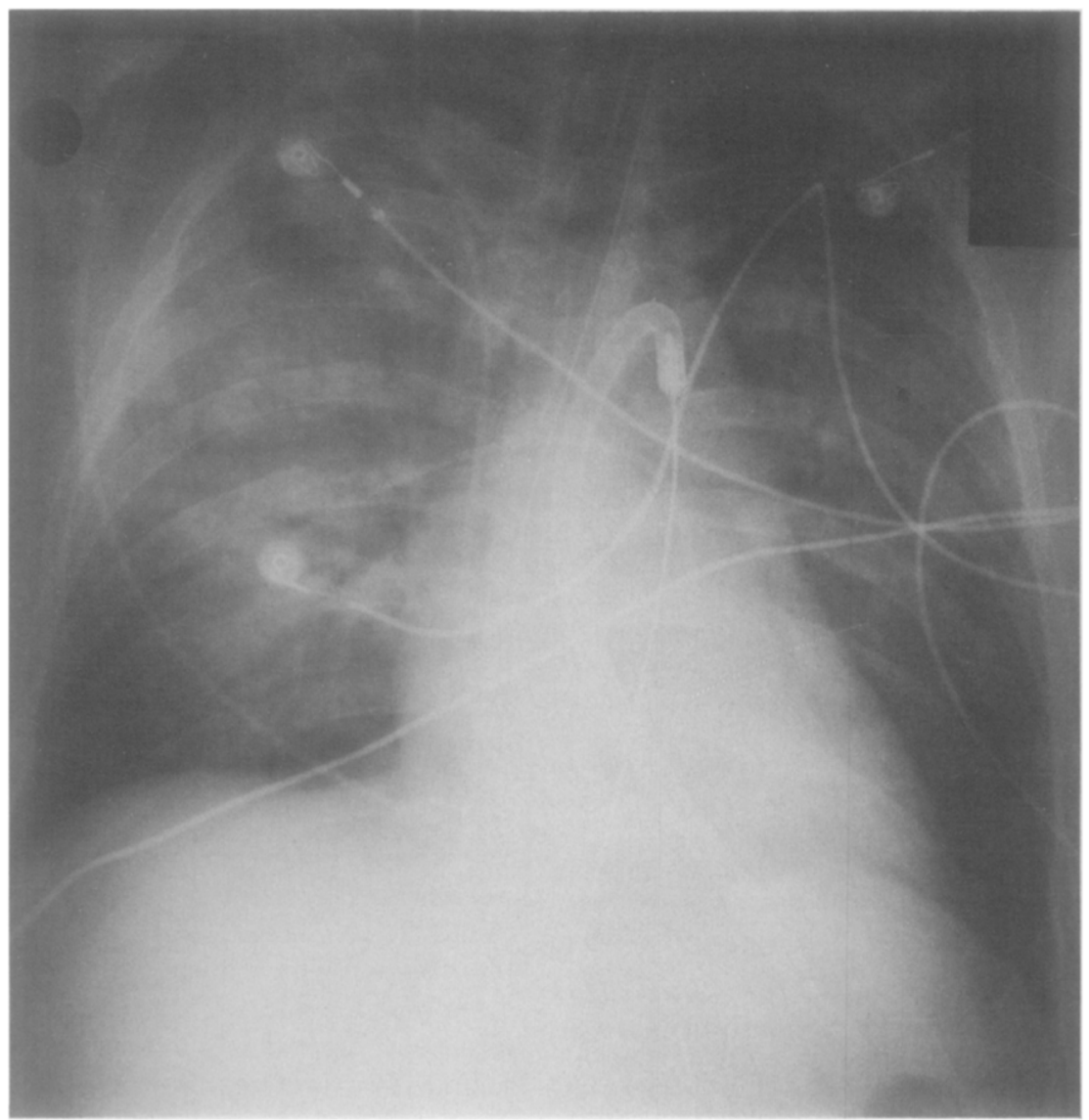

Fig. 1. Anteroposterior chest radiograph of patient (Case No. 1) demonstrating the Hemopump with inflow cannula in the left ventricle and pump housing in the descending aortic arch. The flexible drive shaft couples the pump to the motor outside the patient's body.

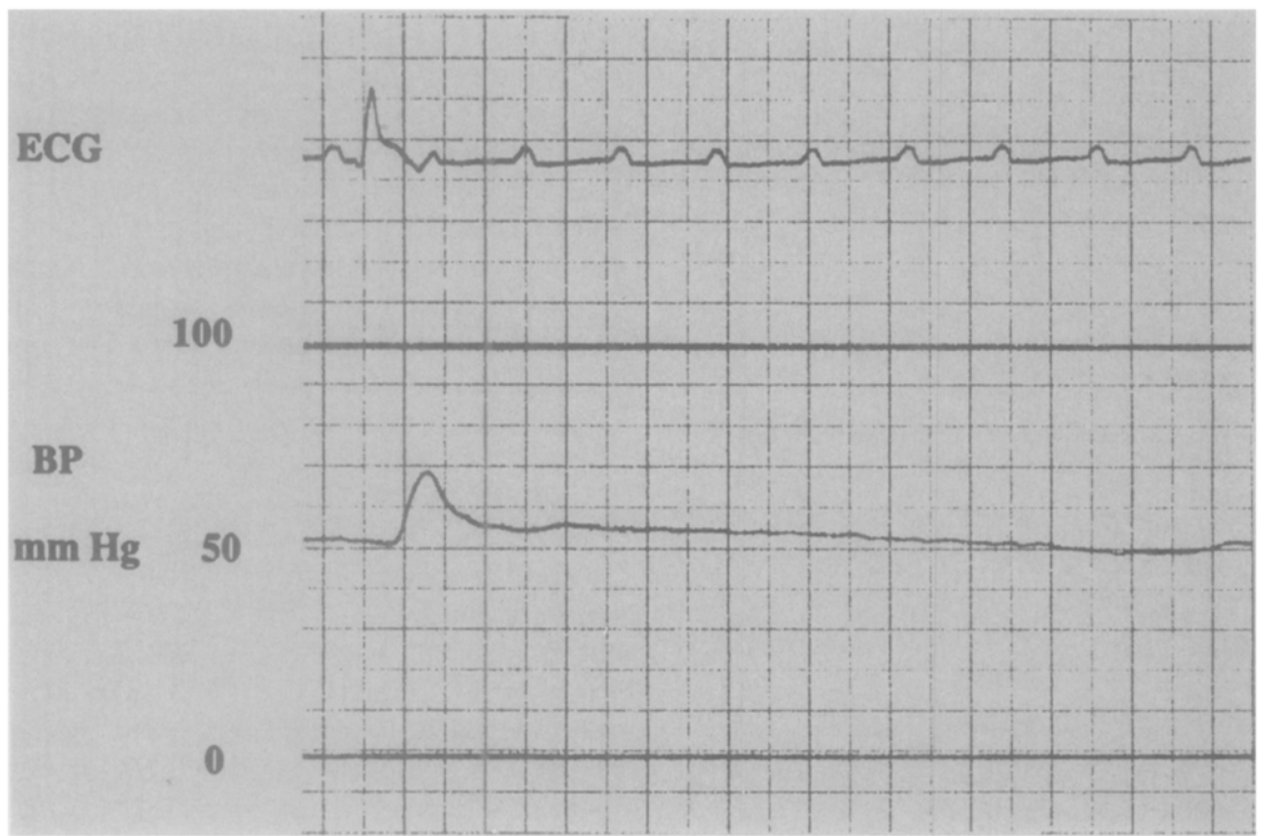

Fig. 2. Simultaneous electrocardiogram and hemodynamic recording demonstrating complete atrioventricular block with a ventricular escape rhythm of 5 to 10 beats $/ \mathrm{min}$ (Case No. 1). Mean aortic pressure was maintained at $50 \mathrm{~mm} \mathrm{Hg}$ by nonpulsatile flow from the Hemopump. 
reducing ventricular distension. During regional ischemia in animal models, the Hemopump increases collateral perfusion within the risk region. ${ }^{6}$ Thus the Hemopump may be especially useful during transient ischemic episodes complicating high-risk coronary angioplasty.

Although the Hemopump has a large $21 \mathrm{~F}$ profile seated across the aortic valve, it did not interfere with routine coronary angiography or angioplasty. The Hemopump produces little hemolysis or aortic valve trauma, and thus may be useful for long-term support. ${ }^{6}$ Nevertheless, the size of the device and the requirement for placement via surgical arteriotomy of the femoral artery limit the speed of insertion. Furthermore, placement of the distal catheter in the left ventricular cavity can mechanically induce ventricular arrhythmias or dislodge mural thrombus. These preliminary findings suggest that circulatory assistance using the Nimbus Hemopump may be useful in high-risk patients undergoing coronary angioplasty. The long-term effect of this device on survival in patients with cardiogenic shock is currently under study.

\section{REFERENCES}

1. Lee L, Bates ER, Pitt B, et al. Percutaneous transluminal coronary angioplasty improves survival in acute myocardial in farction complicated by cardiogenic shock. Circulation 1988;78:1345-51.

2. Shawl FA, Domanski MJ, Punja S, Hernandez TJ. Percutaneous cardiopulmonary bypass supported in high-risk patients undergoing percutaneous transluminal coronary angioplasty. Am J Cardiol 1989;64:1258-63.

3. Alcan K, Stertzer S, Walsh J, et al. The role of intra-aortic balloon counterpulsation in patients undergoing percutaneous transluminal coronary angioplasty. AM HEART J 1983; 105:52730 .

4. Vogel RA. The Maryland Experience: angioplasty and valvuloplasty using percutancous cardiopulmonary support. Am J Cardiol 1988;62:11K-4K.

5. Phillips SJ, Zeff PH, Kongtahworn C, et al. Percutaneous cardiopulmonary bypass: application and indication for use. Ann Thorac Surg 1989;47:121-3.

6. Merhige ME, Smalling RW, Cassidy D, et al. Effect of the Hemopump left ventricular assist device on regional myocardial perfusion and function: reduction of ischemia during coronary occlusion. Circulation 1989;80:III-158-66.

\section{Delayed complete heart block complicating percutaneous transluminal coronary angioplasty}

R. Andrew Rauh, MD, Dale G. Senior, MD, and William P. Miller, MD. Madison, Wisc.

From the Cardiology Section, Department of Medicine, University of Wisconsin.

Reprint requests: R.A. Rauh, MD, Cardiology Section, H6/349, University of Wisconsin, 600 Highland Ave., Madison, WI 53792.

$\mathbf{4} / \mathbf{4} / \mathbf{2 2 9 1 3}$
Routine, temporary pacemaker insertion prior to percutaneous transluminal coronary angioplasty (PTCA) is a common practice to prevent asystole and symptomatic bradycardia during balloon inflation involving atherosclerotic lesions of the right and circumflex coronary arteries. ${ }^{1}$ These rhythm abnormalities are usually transient, resolving with balloon deflation. New conduction defects following PTCA do occur, but they are infrequent, occurring in only $0.9 \%$ of National Heart, Lung, and Blood Institute (NHLBI) PTCA registry patients ${ }^{2}$ and in $1.4 \%$ of the patients undergoing PTCA at Emory University. ${ }^{3}$ In these two reports, the majority of these conduction defects resolved during the hospitalization, with none progressing to complete heart block requiring a permanent pacemaker. We describe a patient who developed delayed complete heart block 30 hours following PTCA complicated by side branch occlusion.

The patient was a 68-year-old man with a history of smoking, hypertension, and elevated cholesterol who presented with a 10-day history of progressive angina with electrocardiographic (ECG) changes consistent with anterior ischemia (Fig. 1, A). He was hospitalized and started on medical therapy, with resolution of his chest pain. Serial cardiac enzyme blood samples for myocardial infarction (MI) were negative. He underwent coronary angiography the day following admission, revealing a $95 \%$ mid-left anterior descending (LAD) lesion. There was nonocclusive disease in the right and circumflex coronary arteries. $\mathrm{He}$ remained asymptomatic with medical therapy and underwent PTCA of the LAD lesion on the sixth hospital day. The procedure was performed with a $3 \mathrm{~mm}$ Profile Plus balloon and 0.014 flexible steerable guide wire (USCI Division of C. R. Bard, Billerica, Mass.). The initial gradient across the LAD lesion was $44 \mathrm{~mm}$ hg. The balloon catheter was positioned distal to the first septal perforator and two inflations each at $8 \mathrm{~atm}$ for 60 seconds were performed. The postdilatation gradient was $2 \mathrm{~mm} \mathrm{Hg}$. Final angiography following balloon catheter removal showed that the stenosis had been reduced from $95 \%$ to less than $25 \%$. A septal branch arising just prior to and not involved in the stenotic site was totally occluded (Fig. 2, $A$ and $B$ ). The patient experienced chest pain following PTCA but repeat injection of the left coronary system continued to show the LAD to be patent and the first septal perforator to still be occluded. The patient's chest pain resolved with intravenous nitrates. An ECG performed 1 hour following the procedure (Fig. 1, B) showed a new right bundle branch block (RBBB). Cardiac enzyme values drawn serially over the next 24 hours were elevated, with a peak creatine kinase of $1006 \mathrm{U} / \mathrm{L}$ (normal 0 to $200 \mathrm{U} / \mathrm{L}$ ) and a $14 \% \mathrm{MB}$ fraction (normal $0 \%$ to $3 \%$ ). An ECG the following morning (24 hours after PTCA) showed an RBBB and left anterior fascicular block (LAFB) (Fig. 1,C). The patient remained asymptomatic until approximately 1 PM the day after PTCA, when he developed lightheadedness associated with a pulse rate of 33 beats $/ \mathrm{min}$ and systolic blood pressure of $90 \mathrm{~mm}$ Hg. The ECG (Fig. 1,D)) at this time showed complete 